\title{
Green tea with high-density catechins improves liver function and fat infiltration in non-alcoholic fatty liver disease (NAFLD) patients: A double-blind placebo-controlled study
}

\author{
RYUICHIRO SAKATA ${ }^{1}$, TORU NAKAMURA ${ }^{1,2}$, TAKUJI TORIMURA ${ }^{1,2}$, TAKATO UENO $^{2,3}$ and MICHIO SATA ${ }^{1,2}$ \\ ${ }^{1}$ Division of Gastroenterology, Department of Medicine, Kurume University School of Medicine; \\ ${ }^{2}$ Liver Cancer Division, Research Center for Innovative Cancer Therapy, Kurume University, Kurume 830-0011; \\ ${ }^{3}$ Asakura Medical Association Hospital, Asakura 838-0069, Japan
}

Received May 16, 2013; Accepted August 19, 2013

DOI: 10.3892/ijmm.2013.1503

\begin{abstract}
Catechins, a major component of green tea extract, have anti-hyperlipidemic effects. The present study investigated the effects of consumption of green tea with high-density catechins in non-alcoholic fatty liver disease (NAFLD) patients. Seventeen patients with NAFLD consumed green tea with high-density catechins, low-density catechins or a placebo for 12 weeks in a randomized double-blind study. Ultrasonography and computed tomography (CT) were performed at baseline and after 12 weeks. Serum alanine aminotransferase (ALT) levels and urine 8-isoprostane were monitored and compared to baseline at 4, 8 and 12 weeks. Body fat was significantly decreased in the high-density catechin group compared with the placebo and low-density catechin groups after 12 weeks of consumption. All the patients in the high-density catechin group showed a significantly improved liver-to-spleen CT attenuation ratio compared with the placebo and low-density catechin groups after 12 weeks of consumption. The highdensity catechin group significantly decreased serum ALT levels and reduced urinary 8-isoprostane excretion compared with the placebo and low-density catechin group after 12 weeks of consumption. Based on a reduced proportion of body fat as estimated by bioimpedance measurement, increased liverto-spleen CT attenuation ratio, decreased serum ALT levels
\end{abstract}

Correspondence to: Dr Toru Nakamura, Division of Gastroenterology, Department of Medicine, Kurume University School of Medicine, 67 Asahi-machi, Kurume, Fukuoka 830-0011, Japan

E-mail:ntoru@med.kurume-u.ac.jp

Abbreviations: ALT, alanine aminotransferase; BMI, body mass index; CT, computed tomography; EGCG, epigallocatechin gallate; HCC, hepatocellular carcinoma; NAFLD, non-alcoholic fatty liver disease; NASH, non-alcoholic steatohepatitis

Key words: fatty liver, non-alcoholic fatty liver disease, non-alcoholic steatohepatitis, metabolic syndrome, flavonoid, oxidative stress and reduced urinary 8-isoprostane excretion, we concluded that 12 weeks of $700 \mathrm{ml}$ per day of green tea containing $>1 \mathrm{~g}$ catechin improved liver fat content and inflammation by reducing oxidative stress in patients with NAFLD.

\section{Introduction}

Non-alcoholic fatty liver disease (NAFLD) is one of the most prevalent types of liver diseases. NAFLD prevalence has increased with the change in eating habits, thus identifying effective treatment for NAFLD is a significant public health objective. Lifestyle-related factors such as poor diet, obesity, excessive alcohol intake, diabetes and hyperlipidemia have all been proposed to contribute to NAFLD. In addition to the development of a fatty liver, NAFLD patients may also exhibit inflammation, necrosis and fibrosis of the liver, which are known as non-alcoholic steatohepatitis (NASH) (1). This disease may progress to cirrhosis of the liver and hepatocellular carcinoma (HCC). Lifestyle interventions such as improvement of eating habits or physical activity are commonly recommended for NAFLD and NASH, but no effective medical therapy for these diseases has been established although many medications for the treatment of NAFLD are undergoing clinical trials in the Western countries.

Green tea contains high levels of flavonoids, which have antioxidant properties. Catechin, one of the main flavonoids in green tea, has recently attracted attention for its antitumor and anti-arteriosclerotic effects. Catechins account for $\sim 20 \%$ of the flavonoids in green tea leaves. They have been found to decrease oxidative stress (2) and to exert anti-virus $(3,4)$, anti-thrombotic (5), anti-allergenic (6), anticancer (7), anti-hypertensive (8) and anti-hyperglycemic effects $(9,10)$. In addition, results of animal experiments have indicated that catechins affect the lipid metabolism by decreasing triglyceride and total cholesterol levels (11) and enhancing energy utilization (12). However, the effect of the green tea with high-density catechins on humans and its detailed mechanism have yet to be clarified. To the best of our knowledge, this is the first study to examine the effects of green tea containing high-density catechins on NAFLD in humans. We report herein the results of a double-blind, controlled study examining the effects of green tea containing 
high-density catechins and a catechin-free green tea-flavored control beverage on liver function and fatty liver status in NAFLD patients. We found that NAFLD patients consuming green tea with high-density catechins for 12 weeks showed improved liver function and reduced liver fat deposition. In addition, we examined the safety of consuming green tea with high-density catechins for NAFLD patients.

\section{Materials and methods}

Subject selection. In total, 17 NAFLD patients ( 7 men and 10 women), aged 20-70 years, were included in this randomized, double-blind, controlled, investigator-initiated trial. The exclusion criteria were the presence of severe acute or chronic diseases (liver, heart or renal failure), infectious or autoimmune liver diseases (positive for hepatitis B surface antigens, anti-hepatitis $\mathrm{C}$ virus, anti-nuclear or anti-mitochondrial antibodies), known allergies to compounds of tea or polyphenol-rich food, acute infectious diseases, diseases involving systemic inflammation, participation in another study within the last month, alcohol abuse and the use of concomitant supplements. Green tea adjusted to $1,080 \mathrm{mg} /$ $700 \mathrm{ml}$ or $200 \mathrm{mg} / 700 \mathrm{ml}$ catechin content and green tea-flavored beverage $(0 \mathrm{mg} / 700 \mathrm{ml}$ catechin content $)$ were prepared by Kao Corporation (Tokyo, Japan). The beverages were packaged in $350 \mathrm{ml}$ steel cans with an identical appearance and distributed to patients by courier. An independent investigator performed subject randomization. All the patients provided informed written consent to participate in the study. The study was performed at the Kurume University Hospital and the study protocol conformed to the ethics guidelines of the 1975 Helsinki Declaration, as reflected in prior approval by the institutional Ethics Committee of the Kurume University School of Medicine.

Experimental protocol. Each patient was instructed to refrain from eating flavonoid-rich foods and supplements during the trial and was then instructed to fast for $8 \mathrm{~h}$ before undergoing the examinations, which were always performed in the morning. These included clinical examination (height, weight and body fat percentage), blood and urine sampling and routine abdominal ultrasonography. Body mass index (BMI) was calculated as weight in kilograms divided by the square of height in meters. The rate of body fat was estimated by bioelectrical impedance analysis performed using InnerScan ${ }^{\circledR}$ (Tanita BC-511, Tanita Corporation, Tokyo, Japan) $(13,14)$. The green tea beverages were consumed for 12 weeks. During this period, patients underwent follow-up examinations in the fourth, eighth and twelfth week. Additional follow-up data were collected four weeks after the end of the green tea consumption. Quantitative abdominal computerized tomography (CT) was performed at the beginning and the end of the study period.

Green teas and placebo (control). Patients were randomized to consume one of the three types of green tea for 12 weeks. Green tea containing $1,080 \mathrm{mg} / 700 \mathrm{ml}$ or $200 \mathrm{mg} / 700 \mathrm{ml}$ catechins and green tea-flavored beverage $(0 \mathrm{mg} / 700 \mathrm{ml}$ catechins) were prepared by Kao Corporation (Tokyo, Japan). Patients consumed $700 \mathrm{ml}$ of green tea every day with meals. The tea containing $200 \mathrm{mg}$ catechins per $700 \mathrm{ml}$ was similar in catechin contents to most commercially available green teas. The intake quantity of $700 \mathrm{ml}$ per day is typical of Japanese tea intake. Caffeine content, another component of green tea, was normalized in all three teas to $120 \mathrm{mg}$ per $700 \mathrm{ml}$.

Quantitative abdominal CT. Abdominal CT was performed to determine the size of each patient's liver and spleen. Findings of many reports have shown that CT liver attenuation corrected for spleen attenuation allows more accurate evaluations of the pathological hepatosteatosis (15-17). Therefore we measured liver attenuation at five sites by CT, one in each hepatic segment from segment II to segment VIII (Couinaud classification), in order to calculate the average liver attenuation. Similarly we also measured spleen attenuation at five sites and calculated the average spleen attenuation. The ratio of liver to spleen attenuation was then calculated and values were compared before and after green tea consumption.

Biomarkers of oxidative stress. Recently, 8-isoprostane (prostaglandin $\mathrm{F} 2 \alpha$ ) has attracted attention as an in vivo indicator of oxidative stress due to its relative stability among prostaglandin isomers $(18,19)$. Urine 8-isoprostane was measured using an EIA kit (Cayman Chemical Company, MI, United States). This assay was based on competition between 8-isoprostane and an 8-isoprostane-acetylcholinesterase conjugate (8-isoprostane tracer) for a limited number of 8 -isoprostane-specific rabbit anti-serum binding sites. As the concentration of the 8 -isoprostane tracer remained constant while the concentration of 8-isoprostane varied, the amount of 8-isoprostane tracer that was able to bind to the rabbit anti-serum was inversely proportional to the concentration of 8-isoprostane in the well. This rabbit anti-serum-8-isoprostane complex bound to a mouse monoclonal anti-rabbit IgG antibody that was also attached to the well. The plate was washed to remove any unbound reagents and then acetylcholinesterase substrate was added to the well. The product of this enzymatic reaction had a distinct yellow color and was absorbed strongly at $412 \mathrm{~nm}$. The intensity of this color, determined spectrophotometrically, was proportional to the amount of 8-isoprostane tracer bound to the well. Data were corrected for urine creatinine and the urine 8-isoprostane $(\mathrm{pg} / \mathrm{ml}) /$ creatinine $(\mathrm{mg} / \mathrm{ml})$ ratio was expressed as urine 8 -isoprostane ( $\mathrm{pg} / \mathrm{mg}$ creatinine).

Statistical analysis. Data are expressed as means \pm SD. Associations among the three patient groups for baseline characteristics, liver CT attenuation and urine 8-isoprostane were compared using analysis of variance (ANOVA). Within each group, comparisons were made using the Student's paired t-test. $\mathrm{P}<0.05$ was considered statistically significant. Statistical analyses were performed using AIST-ANOVA developed by the National Metrology Institute of Japan (NMIJ) and National Institute of Advanced Industrial Science and Technology (AIST) for statistical analysis.

\section{Results}

Subject demographics. Seventeen patients were included in the present study and were randomized to consume either green tea with high- or low-density catechins or a control beverage with no catechins. Clinical and laboratory characteristics of 
Table I. Baseline clinical characteristics $(n=17)$.

\begin{tabular}{llcc}
\hline Group & $\begin{array}{c}\text { Placebo } \\
(\mathrm{n}=5)\end{array}$ & $\begin{array}{c}\text { Low-density catechins } \\
(\mathrm{n}=5)\end{array}$ & $\begin{array}{c}\text { High-density catechins } \\
(\mathrm{n}=7)\end{array}$ \\
\hline Age (years) & $54.2 \pm 8.1$ & $51.4 \pm 14.8$ & $47.1 \pm 17.2$ \\
Body weight $(\mathrm{kg})$ & $74.1 \pm 18.3$ & $77.6 \pm 13.8$ & $70.7 \pm 13.0$ \\
Body fat $(\%)$ & $36.1 \pm 6.2$ & $36.0 \pm 4.8$ & $34.3 \pm 6.9$ \\
Body mass index $\left(\mathrm{kg} / \mathrm{m}^{2}\right)$ & $30.0 \pm 4.4$ & $29.1 \pm 1.8$ & $28.0 \pm 2.0$
\end{tabular}

Values are presented as means $\pm \mathrm{SD}$. P-value represents the comparison of groups by ANOVA.

Table II. Baseline laboratory characteristics $(n=17)$.

\begin{tabular}{|c|c|c|c|c|}
\hline Group & $\begin{array}{l}\text { Placebo } \\
(n=5)\end{array}$ & $\begin{array}{l}\text { Low-density catechins } \\
\qquad(\mathrm{n}=5)\end{array}$ & $\begin{array}{l}\text { High-density catechins } \\
\qquad(\mathrm{n}=7)\end{array}$ & P-value \\
\hline AST (IU/l) & $110 \pm 58$ & $104 \pm 47$ & $120 \pm 57$ & 0.88 \\
\hline ALT (IU/l) & $94 \pm 50$ & $81 \pm 47$ & $101 \pm 95$ & 0.89 \\
\hline LDH (IU/1) & $225 \pm 39$ & $220 \pm 32$ & $224 \pm 59$ & 0.96 \\
\hline ALP (IU/l) & $198 \pm 75$ & $246 \pm 82$ & $230 \pm 63$ & 0.61 \\
\hline$\gamma$-GTP (IU/l) & $69 \pm 54$ & $74 \pm 44$ & $81 \pm 51$ & 0.33 \\
\hline Ch-E (IU/l) & $194 \pm 24$ & $201 \pm 32$ & $191 \pm 42$ & 0.53 \\
\hline Total protein $(\mathrm{g} / \mathrm{dl})$ & $7.9 \pm 1.0$ & $7.5 \pm 0.9$ & $7.1 \pm 0.7$ & 0.20 \\
\hline Total bilirubin $(\mathrm{mg} / \mathrm{dl})$ & $0.8 \pm 0.4$ & $0.9 \pm 0.3$ & $0.9 \pm 0.3$ & 0.70 \\
\hline BUN (mg/dl) & $10.1 \pm 5.0$ & $9.5 \pm 3.8$ & $11.0 \pm 3.4$ & 0.51 \\
\hline Creatinine (mg/dl) & $0.9 \pm 0.4$ & $1.0 \pm 0.3$ & $1.1 \pm 0.2$ & 0.64 \\
\hline Total cholesterol (mg/dl) & $161 \pm 59$ & $197 \pm 33$ & $189 \pm 55$ & 0.61 \\
\hline Glucose (mg/dl) & $97 \pm 18$ & $99 \pm 21$ & $94 \pm 19$ & 0.18 \\
\hline Hemoglobin $(\mathrm{g} / \mathrm{dl})$ & $12.8 \pm 1.2$ & $14.1 \pm 2.4$ & $11.5 \pm 1.9$ & 0.56 \\
\hline Erythrocytes $\left(10^{4} / \mu 1\right)$ & $390 \pm 41$ & $411 \pm 60$ & $405 \pm 55$ & 0.21 \\
\hline $\operatorname{MCV}(f l)$ & $90.3 \pm 7.0$ & $89.5 \pm 8.1$ & $91.2 \pm 9.4$ & 0.87 \\
\hline $\mathrm{MCH}(\mathrm{pg})$ & $31.4 \pm 3.0$ & $30.8 \pm 2.5$ & $32.5 \pm 2.9$ & 0.90 \\
\hline Thrombocytes $\left(10^{4} / \mu \mathrm{l}\right)$ & $19.5 \pm 5.5$ & $17.9 \pm 6.8$ & $18.6 \pm 4.9$ & 0.84 \\
\hline Leukocytes $\left(10^{3} / \mu 1\right)$ & $6.3 \pm 2.1$ & $5.2 \pm 2.9$ & $6.2 \pm 1.9$ & 0.87 \\
\hline
\end{tabular}

Values are presented as means \pm SD. P-value represents the comparison of groups by ANOVA. AST, aspartate aminotransferase; ALT, alanine aminotransferase; LDH, lactate dehydrogenase; ALP, alkaline phosphatase; $\gamma$-GTP, $\gamma$-glutamyl transferase; Ch-E, cholinesterase; BUN, blood urea nitrogen; $\mathrm{MCV}$, mean corpuscular volume; $\mathrm{MCH}$, mean corpuscular hemoglobin.

the study population are presented in Tables I and II, respectively. There were no significant differences among the three groups for clinical characteristics at baseline, including age, body weight, body fat percentage or BMI (Table I). There were also no significant differences among the three groups in laboratory data at baseline (Table II).

Reduction of body fat percentage in the high-density catechin group. Comparison of the data at baseline and 12 weeks later showed that the largest decrease in body weight occurred in the high-density catechin group. However, there was no significant difference in the percentage change of body weight among the three groups (high-density catechins, $-3.8 \pm 2.7 \%$; low-density catechins, $-0.9 \pm 3.5 \%$; placebo, $-1.4 \pm 3.7 \%$; Fig. 1 ).
BMI also decreased after 12 weeks of tea consumption, but there was no significant difference among the three groups (high-density catechins, $-3.3 \pm 1.9 \%$; low-density catechins, $-0.5 \pm 2.0 \%$; placebo, $-1.2 \pm 3.9 \%$; Fig. 2).

Body fat percentage decreased significantly from $34.3 \pm 6.9 \%$ (baseline) to $31.8 \pm 6.0 \%$ after 12 weeks of high-density catechin tea consumption $(\mathrm{P}<0.05)$. Body fat percentage decreased significantly more in the high-density catechin group $(-7.3 \pm 2.2 \%)$ than in the placebo $(0.9 \pm 2.1 \%)$ and low-density catechin (-0.6 $\pm 2.4 \%$ ) groups after 12 weeks (Fig. 3).

The liver-to-spleen $\mathrm{CT}$ attenuation ratio increased from $91.8 \pm 4.6 \%$ (baseline) to $101.8 \pm 4.7 \%$ after 12 weeks of consumption of high-density catechin tea. The liver-to-spleen $\mathrm{CT}$ attenuation ratio showed greater improvement in all 


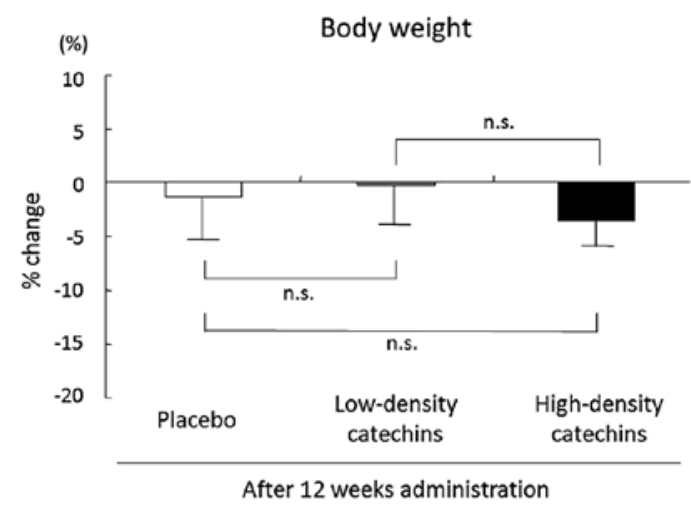

Figure 1. Percentage change of body weight after 12 weeks of catechin consumption. At 12 weeks, the body weight tended to be decreased in the high-density catechin group. However, there was no significant difference among the three groups. n.s., not statistically significant.

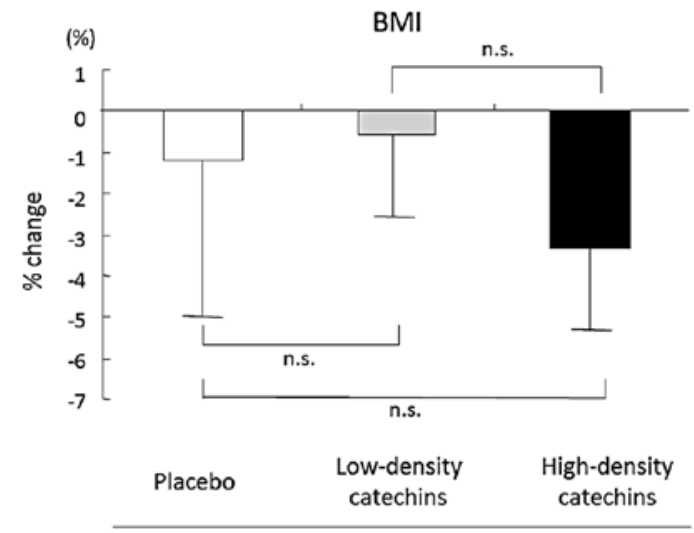

After 12 weeks administration

Figure 2. Percentage change of body mass index (BMI) after 12 weeks of catechin consumption. BMI decreased after 12 weeks of catechin consumption, but there was no statistically significant difference among the three groups. BMI, body mass index; n.s., not statistically significant.

patients in the high-density catechin group $(11.3 \pm 2.8 \%)$ than in the placebo $(-3.3 \pm 8.5 \%)$ and low-density catechin $(-6.1 \pm 12.1 \%)$ groups (Fig. 4).

Improvement of serum alanine aminotransferase (ALT) levels by high-density catechin treatment. Serum ALT is an important marker of liver inflammation. Percentage change in serum ALT levels was significantly more negative in the high-density catechin group $(-42.1 \pm 11.3 \%)$ than in the placebo $(-3.1 \pm 7.8 \%)$ and low-density catechin $(0.5 \pm 5.1 \%)$ groups after 12 weeks of consumption of high-density catechin tea (Fig. 5).

Reduction of urinary 8-isoprostane excretion by high-density catechin treatment. Urine 8-isoprostane is a specific marker of oxidative stress. Urine 8-isoprostane excretion was reduced from $249.6 \pm 11.6 \mathrm{pg}$ (baseline) to $172.0 \pm 9.0 \mathrm{pg}$ after 12 weeks of high-density catechin tea consumption. The percentage change in 8-isoprostane levels was worse in the high-density catechin group $(-31.1 \pm 9.0 \%)$ than in the placebo $(-1.7 \pm 9.1 \%)$ or low-density catechin (2.1 $\pm 6.1 \%$ ) groups (Fig. 6$)$.

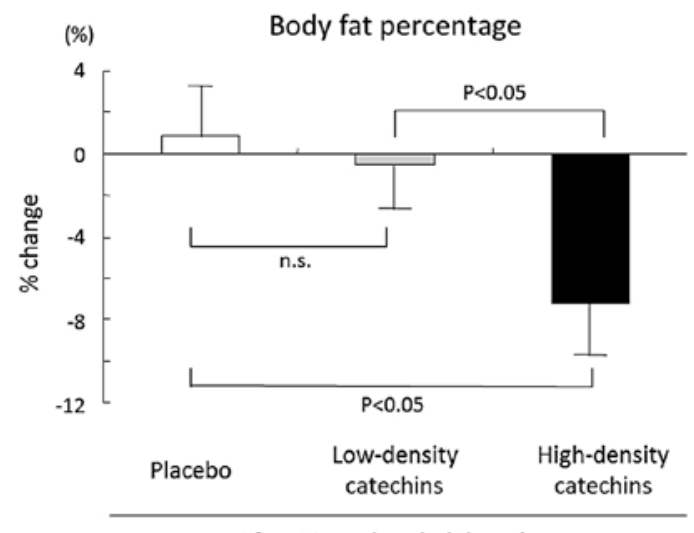

After 12 weeks administration

Figure 3. Percentage change of body fat percentage after 12 weeks of catechin consumption. Body fat decreased significantly more in the high-density catechin group than in the placebo and low-density catechin groups after 12 weeks of tea consumption $(\mathrm{P}<0.05)$. n.s., not statistically significant.

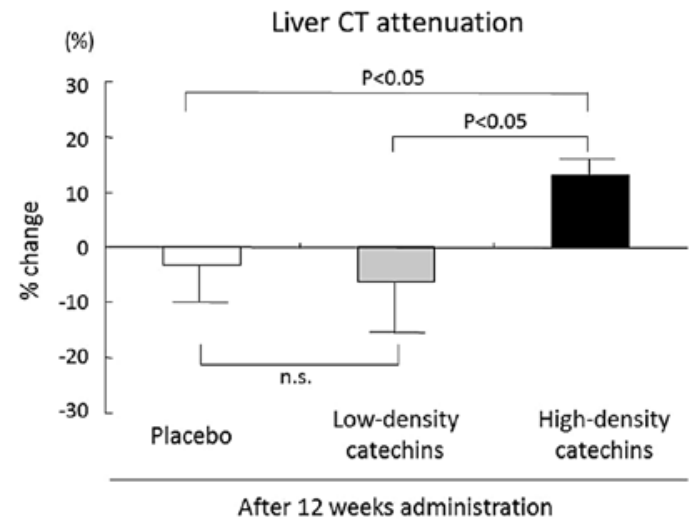

Figure 4. Percentage change of liver-to-spleen $\mathrm{CT}$ attenuation ratio after 12 weeks of catechin consumption. The liver-to-spleen CT attenuation ratio was significantly improved in all patients in the high-density catechin group relative to those in the placebo and low-density catechin groups after 12 weeks of tea consumption $(\mathrm{P}<0.05)$. n.s., not statistically significant.

\section{Discussion}

NAFLD is a prevalent disease that is detected by medical examination and ultrasonography. Among NAFLD categories, patients with NASH, which is similar to alcoholic steatohepatitis in terms of pathological findings, have a poor prognosis (20). The lesions most commonly accepted with NASH include steatosis, ballooning degeneration of hepatocyte, mild diffuse lobular mixed acute and chronic inflammation and perivenular and perisinusoidal collagen deposition. Mallory's hyaline, vacuolated nuclei in periportal hepatocytes, lobular lipogranulomas and PAS-diastaseresistant Kupffer cells are also common in NASH. NASH may be an underlying cause of cryptogenic cirrhosis $(21,22)$. The worldwide epidemic of obesity has increased the awareness of NAFLD from that of a curiosity to one of a potentially progressive liver disease that increases the risk of cirrhosis and HCC (23). A report by Marrero et al (24) indicated that cryptogenic liver disease is a common etiology of diseases in patients with HCC. NAFLD has been reported to account for 


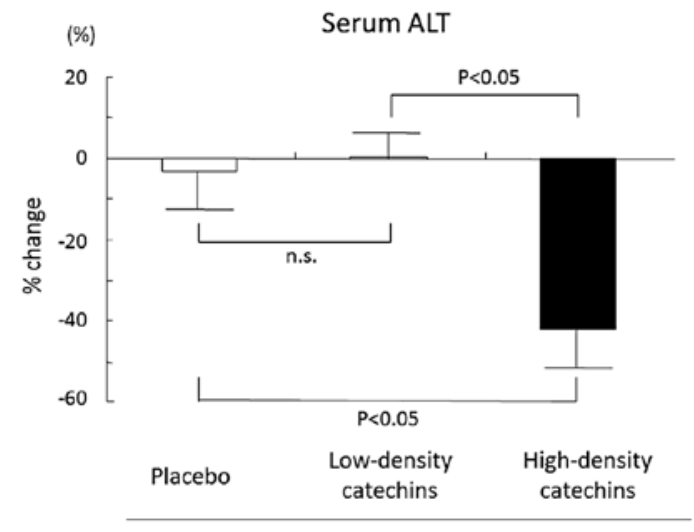

After 12 weeks administration

Figure 5. Percentage change of serum ALT values after 12 weeks of catechin consumption. Percentage change of serum ALT values was significantly decreased in the high-density catechin group compared with the placebo and low-density catechin groups after 12 weeks of catechin consumption $(\mathrm{P}<0.05)$. n.s., not statistically significant.

at least $13 \%$ of HCC cases (24). Cryptogenic cirrhosis patients were found to have higher plasma levels of glucose, cholesterol and triglyceride, all parameters of insulin resistance (22). Obesity is an independent risk factor for HCC in patients with cryptogenic cirrhosis (25). Not all cases of NAFLD progress to cirrhosis and liver cancer. Early diagnosis and treatment of NAFLD may prevent progression to cirrhosis. As reported above, the improvement of eating habits is necessary to improve hyperlipidemia, insulin resistance and obesity.

As for whether green tea is effective for the improvement of insulin resistance and hyperlipidemia when consumed with a meal, the present study indicates that it is effective for treatment in NAFLD. It is believed that dietary therapy is preferable to medical therapy for the treatment of NAFLD, considering the mechanism of onset, but lifestyle changes can be difficult to implement. Thus, the development of an effective medical therapy is necessary. Epigallocatechin gallate (EGCG), the main catechin in green tea, is believed to reduce liver oxidation stress. The components of NAFLD have not yet been fully elucidated, but the following steps are considered to be the main mechanism. Free fatty acids are absorbed by the liver through the intestinal tract after a meal and are oxidized by mitochondria and peroxisomes. If fatty acid uptake by hepatocytes increases, fatty acid pools in the liver increase and accumulate in the hepatocytes as acylglycerol, increasing the load on hepatic mitochondria. Fatty acids that are not metabolized by mitochondria undergo $\omega$ or $\beta$ oxidation by microsomes or peroxisomes. If a large quantity of fatty acids continues to be deposited in the liver, accumulation of acylglycerol in the hepatocytes induces oxidative stress that may progress to NAFLD (26). It is thought that EGCG reduces oxidative stress in hepatocytes through its potent antioxidant activity. Our study showed that the group consuming 1,080 mg of catechins per day had significantly lower levels of urine 8 -isoprostane, a marker of oxidative stress, at the end of the study than at baseline. The low-density catechin and placebo groups did not show decreased oxidative stress, suggesting that it is necessary to consume $\sim 1 \mathrm{~g}$ of catechins every day to reduce oxidative stress.

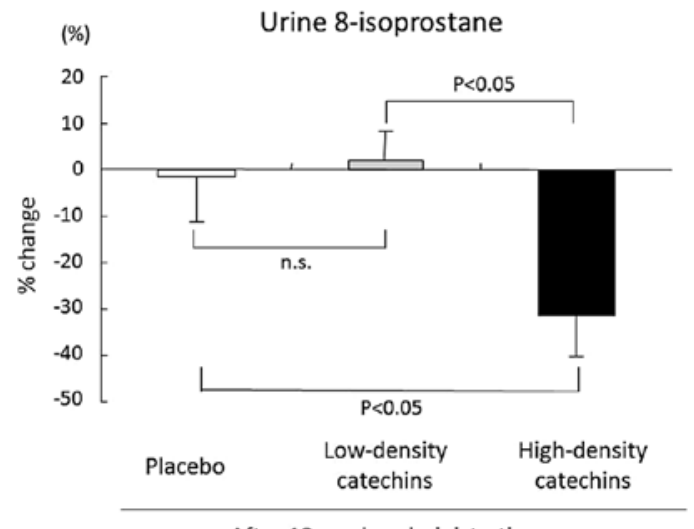

After 12 weeks administration

Figure 6. Percentage change of a marker of oxidative stress after 12 weeks of catechin consumption. Percentage change of urine 8-isoprostane was significantly more negative in the high-density catechin group than in the placebo and low-density catechin groups after 12 weeks of catechin consumption $(\mathrm{P}<0.05)$. n.s., not statistically significant.

Catechins have inhibition effects on lipase, an enzyme related to glucose and fat absorption. EGCG shows inhibitory activity against lipase at a concentration of $0.349 \mu \mathrm{M}$ (IC50) (27). In addition, catechins are reported to have inhibitory effects on $\alpha$-amylase and $\alpha$-glucosidase $(27,28)$. A clinical study of hypertriacylglycerolaemia showed that the increase of triacylglycerol levels in the plasma after oral administration of butter was blunted by $\sim 29 \%$ in response to catechin consumption (29). If fat absorption in the intestinal tract is decreased, liver fatty acid uptake also decreases, which may help prevent the onset of NAFLD.

It has been shown that catechins promote lipid metabolism in the liver (30). Body weight and adiposity were blunted by catechin administration in the obese mouse model C57BL/6J. Increased mRNA expression of acyl-CoA oxidase (ACO), one of the peroxisomal $\beta$-oxidizing enzymes and medium-chain acyl-CoA dehydrogenase (MCAD), a mitochondrial $\beta$-oxidizing enzyme, was observed in the liver of the catechin administration group. Increased hepatocellular mitochondrial $\beta$-oxidation activity promotes the breakdown of fatty acids and it is thought that it acts as a protective mechanism against NAFLD.

Catechins are a natural iron chelator and also serve to influence internal absorption of iron. A controlled study looking at the effects of EGCG on non-heme iron absorption showed that it was decreased by $27 \%$ in patients consuming $300 \mathrm{mg}$ EGCG compared with controls consuming placebo (31). Reports on NASH patients showed that elevated iron stores, iron absorption in the liver (32) and serum ALT levels were decreased by bloodletting treatment (33). Restricting iron absorption through catechins may therefore be effective treatment for NAFLD.

NAFLD is a widespread disease and some cases of NAFLD progress to NASH. It is thought that the existence of steatosis and hepatitis is crucial for a diagnosis of NASH, which can be confirmed by a liver biopsy. Liver biopsy is the golden standard for NASH diagnosis. However, many patients without symptoms who present abnormal serum data, suggesting the presence of NAFLD, do not undergo a liver biopsy. For the present study, we used ultrasonography and X-ray CT to monitor NAFLD as these methods are non-invasive and 
follow-up data can be collected. Blood biochemistry was used for the determination of hepatitis and steatosis status (34). The present study included only patients who had been diagnosed with NAFLD by a specialist. We instructed some participants to consume tea containing five times as much catechin content as normal tea for 12 weeks and did not observe any negative side effects in this group.

The mechanism of onset of NAFLD and NASH has still not been fully elucidated (35). The mechanism by which ingestion of catechins decreases fat accumulation in the liver has also not been determined. Liver fat was decreased along with an oxidative stress marker in response to the consumption of a high catechin tea. Liver inflammation and blood biochemistry also improved in this group. Findings of this study suggest that catechins are useful for the treatment of NAFLD.

\section{Acknowledgements}

The study was supported, in part, by the Japan National Science Foundation (grant no. 16590651). All the tea was provided by Kao Corporation, Japan. However, Kao Corporation was not involved in the funding of or in any part of the study. Part of this study was presented at the 41st annual meeting of the European Association for the Study of the Liver.

\section{References}

1. Neuschwander-Tetri BA and Caldwell SH: Nonalcoholic steatohepatitis: summary of an AASLD single topic conference. Hepatology 38: 1202-1219, 2003.

2. Hakim IA, Harris RB, Brown S, et al: Effect of increased tea consumption on oxidative DNA damage among smokers: a randomized controlled study. J Nutr 133: 3303S-3309S, 2003.

3. Hamza A and Zhan CG: How can (-)-epigallocatechin gallate from green tea prevent HIV-1 infection? Mechanistic insights from computational modeling and the implication for rational design of anti-HIV-1 entry inhibitors. J Phys Chem B 110 2910-2917, 2006.

4. Xu J, Wang J, Deng F, Hu Z and Wang H: Green tea extract and its major component epigallocatechin gallate inhibits hepatitis $\mathrm{B}$ virus in vitro. Antiviral Res 78: 242-249, 2008.

5. Stangl V, Lorenz M and Stangl K: The role of tea and tea flavonoids in cardiovascular health. Mol Nutr Food Res 50: 218-228, 2006.

6. Maeda-Yamamoto M, Inagaki N, Kitaura J, et al: O-methylated catechins from tea leaves inhibit multiple protein kinases in mast cells. J Immunol 172: 4486-4492, 2004.

7. Khan $\mathrm{N}$ and Mukhtar H: Multitargeted therapy of cancer by green tea polyphenols. Cancer Lett 269: 269-280, 2008.

8. Kim JA, Formoso G, Li Y, et al: Epigallocatechin gallate, a green tea polyphenol, mediates NO-dependent vasodilation using signaling pathways in vascular endothelium requiring reactive oxygen species and Fyn. J Biol Chem 282: 13736-13745, 2007.

9. Wolfram S: Effects of green tea and EGCG on cardiovascular and metabolic health. J Am Coll Nutr 26: 373S-388S, 2007.

10. Ueda M, Nishiumi S, Nagayasu H, Fukuda I, Yoshida K and Ashida H: Epigallocatechin gallate promotes GLUT4 translocation in skeletal muscle. Biochem Biophys Res Commun 377: 286-290, 2008.

11. Koo SI and Noh SK: Green tea as inhibitor of the intestinal absorption of lipids: potential mechanism for its lipid-lowering effect. J Nutr Biochem 18: 179-183, 2007.

12. Murase T, Haramizu S, Shimotoyodome A, Tokimitsu I and Hase T: Green tea extract improves running endurance in mice by stimulating lipid utilization during exercise. Am J Physiol Regul Integr Comp Physiol 290: R1550-R1556, 2006.

13. Kyle UG, Genton L, Hans D and Pichard C: Validation of a bioelectrical impedance analysis equation to predict appendicular skeletal muscle mass (ASMM). Clin Nutr 22: 537-543, 2003.
14. Lukaski HC: Applications of bioelectrical impedance analysis: a critical review. Basic Life Sci 55: 365-374, 1990.

15. Duman DG, Celikel C, Tuney D, Imeryuz N, Avsar E and Tozun N: Computed tomography in nonalcoholic fatty liver disease: a useful tool for hepatosteatosis assessment? Dig Dis Sci 51: 346-351, 2006.

16. Church TS, Kuk JL, Ross R, Priest EL, Biltoft E and Blair SN: Association of cardiorespiratory fitness, body mass index, and waist circumference to nonalcoholic fatty liver disease. Gastroenterology 130: 2023-2030, 2006.

17. Oliva MR, Mortele KJ, Segatto E, et al: Computed tomography features of nonalcoholic steatohepatitis with histopathologic correlation. J Comput Assist Tomogr 30: 37-43, 2006.

18. Devaraj S, Hirany SV, Burk RF and Jialal I: Divergence between LDL oxidative susceptibility and urinary $\mathrm{F}(2)$-isoprostanes as measures of oxidative stress in type 2 diabetes. Clin Chem 47: 1974-1979, 2001

19. Patrono $C$ and FitzGerald GA: Isoprostanes: potential markers of oxidant stress in atherothrombotic disease. Arterioscler Thromb Vasc Biol 17: 2309-2315, 1997.

20. Powell EE, Cooksley WG, Hanson R, Searle J, Halliday JW and Powell LW: The natural history of nonalcoholic steatohepatitis: a follow-up study of forty-two patients for up to 21 years. Hepatology 11: 74-80, 1990.

21. Brunt EM: Nonalcoholic steatohepatitis: definition and pathology. Semin Liver Dis 21: 3-16, 2001.

22. Bugianesi E, Leone N, Vanni E, et al: Expanding the natural history of nonalcoholic steatohepatitis: from cryptogenic cirrhosis to hepatocellular carcinoma. Gastroenterology 123: 134-140, 2002.

23. Brunt EM: Nonalcoholic steatohepatitis: pathologic features and differential diagnosis. Semin Diagn Pathol 22: 330-338, 2005.

24. Marrero JA, Fontana RJ, Su GL, Conjeevaram HS, Emick DM and Lok AS: NAFLD may be a common underlying liver disease in patients with hepatocellular carcinoma in the United States. Hepatology 36: 1349-1354, 2002.

25. Caldwell SH, Oelsner DH, Iezzoni JC, Hespenheide EE, Battle EH and Driscoll CJ: Cryptogenic cirrhosis: clinical characterization and risk factors for underlying disease. Hepatology 29: 664-669, 1999.

26. Kersten S, Seydoux J, Peters JM, Gonzalez FJ, Desvergne B and Wahli W: Peroxisome proliferator-activated receptor alpha mediates the adaptive response to fasting. J Clin Invest 103: 1489-1498, 1999.

27. Nakai M, Fukui Y, Asami S, et al: Inhibitory effects of oolong tea polyphenols on pancreatic lipase in vitro. J Agric Food Chem 53: 4593-4598, 2005.

28. Matsui T, Yoshimoto C, Osajima K, Oki T and Osajima Y: In vitro survey of alpha-glucosidase inhibitory food components. Biosci Biotechnol Biochem 60: 2019-2022, 1996.

29. Unno T, Tago M, Suzuki Y, et al: Effect of tea catechins on postprandial plasma lipid responses in human subjects. Br J Nutr 93: 543-547, 2005.

30. Murase T, Nagasawa A, Suzuki J, Hase T and Tokimitsu I: Beneficial effects of tea catechins on diet-induced obesity: stimulation of lipid catabolism in the liver. Int J Obes Relat Metab Disord 26: 1459-1464, 2002.

31. Ullmann U, Haller J, Bakker GC, Brink EJ and Weber P. Epigallocatechin gallate (EGCG) (TEAVIGO) does not impair nonhaem-iron absorption in man. Phytomedicine 12: 410-415, 2005.

32. Kohgo Y, Ohtake T, Ikuta K, Suzuki Y, Torimoto Y and Kato J: Dysregulation of systemic iron metabolism in alcoholic liver diseases. J Gastroenterol Hepatol 23 (Suppl 1): S78-S81, 2008.

33. Sumida Y, Kanemasa K, Fukumoto K, et al: Effect of iron reduction by phlebotomy in Japanese patients with nonalcoholic steatohepatitis: A pilot study. Hepatol Res 36: 315-321, 2006.

34. Chalasani N, Younossi Z, Lavine JE, et al: The diagnosis and management of non-alcoholic fatty liver disease: practice guideline by the American Gastroenterological Association, American Association for the study of liver diseases, and American College of Gastroenterology. Gastroenterology 142: 1592-1609, 2012.

35. Farrell GC, van Rooyen D, Gan L and Chitturi S: NASH is an inflammatory disorder: pathogenic, prognostic and therapeutic implications. Gut Liver 6: 149-171, 2012. 